Research Article

\title{
Averaging Principle for Backward Stochastic Differential Equations
}

\author{
Yuanyuan Jing $(\mathbb{D}$ and Zhi Li $\mathbb{C}$ \\ School of Information and Mathematics, Yangtze University, Jingzhou 434023, China \\ Correspondence should be addressed to Zhi Li; lizhi_csu@126.com
}

Received 10 October 2020; Revised 29 January 2021; Accepted 15 February 2021; Published 4 March 2021

Academic Editor: Nikos I. Karachalios

Copyright ( 92021 Yuanyuan Jing and Zhi Li. This is an open access article distributed under the Creative Commons Attribution License, which permits unrestricted use, distribution, and reproduction in any medium, provided the original work is properly cited.

The averaging principle for BSDEs and one-barrier RBSDEs, with Lipschitz coefficients, is investigated. An averaged BSDEs for the original BSDEs is proposed, as well as the one-barrier RBSDEs, and their solutions are quantitatively compared. Under some appropriate assumptions, the solutions to original systems can be approximated by the solutions to averaged stochastic systems in the sense of mean square.

\section{Introduction}

The backward stochastic differential equations (BSDEs) were first studied by Parduox and Peng in [1] and have the following type:

$$
Y_{t}=\xi+\int_{t}^{T} g\left(s, Y_{s}, Z_{s}\right) \mathrm{d} s-\int_{t}^{T} Z_{s} \mathrm{~d} B_{s},
$$

where $\left\{B_{t}: 0 \leq t \leq T\right\}$ is a $d$-dimensional Brownian motion defined on the probability space $(\Omega, \mathscr{F}, \mathbb{P})$ with the natural filtration $\left\{\mathscr{F}_{t}: 0 \leq t \leq T\right\}$, the terminal value $\xi$ is square integrable, and $g$ is a mapping from $\Omega \times[0, T] \times \mathbb{R} \times \mathbb{R}^{d}$ to $\mathbb{R}$. They proved that equation (1) has a unique adapted and square integrable solution when $g$ is globally Lipschitz. Mao [2] and Wang and Wang [3] both considered the adapted solution to equation (1) when $g$ is non-Lipschitz. Besides, Lepeltier and Martin [4] studied the case of continuous coefficients, while the locally Lipschitz coefficient was investigated by Bahlali in [5].

Since then, in [6], El Karoui et al. began to introduce the notion of a backward stochastic differential equation reflected to one continuous lower barrier (RBSDEs in short). That is, a solution for such an equation associated with a coefficient $g$, a terminal value $\xi$, and a continuous barrier $S_{t}$, is a triple $\left(X_{t}, Y_{t}, Z_{t}\right)_{0 \leq t \leq T}$ of adapted processes valued on $\mathbb{R}^{1+d+1}$, which satisfies a square integrability condition:

$$
\begin{aligned}
Y_{t} & =\xi+\int_{t}^{T} g\left(s, Y_{s}, Z_{s}\right) \mathrm{d} s+K_{T}-K_{t}-\int_{t}^{T} Z_{s} \mathrm{~d} B_{s}, \\
Y_{t} & \geq S_{t}, \\
K_{0} & =0 \\
\int_{0}^{T}\left(Y_{t}-S_{t}\right) \mathrm{d} K_{t} & =0 .
\end{aligned}
$$

They established that this equation has a unique smooth square integrable solution when $g$ is Lipschitz in $y, z$. After that, many scholars have studied the solutions of equation (2) under different conditions, such as Matoussi [7] considered the case of continuous and at most linear growth in $(y, z)$. Hamadene [8] studied the case of a right-continuous with left limits barrier and Lepeltier, and $\mathrm{Xu}$ [9] investigated the case of discontinuous barrier. For the monotonicity, general increasing growth conditions were investigated by Lepeltier et al. [10].

On the contrary, averaging principle, which is usually used to approximate dynamical systems under random fluctuations, has long and rich history in multiscale problems (see, e.g., [11-14]). However, motivated by the above works, the averaging principle for equation (1), even for 
equation (2), has not introduced at all. The main motivation of our work is to seek an answer to the following interesting question: compared with the general stochastic differential equations, do the backward stochastic differential equations have the averaging principle of solutions? So, in this paper, we will consider this issue under Lipschitz conditions. But, due to the characteristics of the equations of BSDEs and RBSEDs with a barrier, we should first consider the relationship among the random variables $Z$ and $Y$ and the function $K_{t}$, which is also one of the most challenging tasks in this paper.

The remaining part of this paper is organized as follows. In Section 2, we present some preliminaries and assumptions for the later use. In Section 3, we investigate the averaging principle for the BSDEs under some proper conditions. Then, the averaging principle for the RBSDEs with a barrier will be given in Section 4. Finally, in Section 5, we design two examples to demonstrate the efficiency of the proposed method.

\section{Preliminaries}

Throughout, this paper $\left\{B_{t}: 0 \leq t \leq T\right\}$ is a $d$-dimensional standard Brownian motion defined on a probability space $\{\Omega, \mathscr{F}, \mathbb{P}\}$. Let $\left\{\mathscr{F}_{t}: 0 \leq t \leq T\right\}$ be the natural filtration of $\left\{B_{t}\right\}$, where $\mathscr{F}_{0}$ contains all $P$-null sets of $\mathscr{F}$ and let $\mathscr{P}$ be the $\sigma$-algebra of predictable subsets of $\Omega \times[0, T]$. In addition, we define the following:

(C1) $L^{2}\left(\Omega, \mathscr{F}_{T}, \mathbb{P}\right):=\left\{\xi: \mathscr{F}_{T}-\right.$

measurable random variable, $\left.\mathbb{E}\left[|\xi|^{2}\right]<\infty\right\}$.

$(C 2) S^{2}(0, T ; \mathbb{R}):=$

$\left\{\begin{array}{c}\varphi: \varphi \text { is continuous processes with values in } \mathbb{R} \text { such that }\|\varphi\|_{S^{2}}^{2}= \\ E\left[\sup _{0 \leq t \leq T}\left|\varphi_{t}\right|^{2}\right]<\infty \text {, and } \varphi_{t} \text { is } \mathscr{F}_{t}-\text { measurable, for any } t \in[0, T]\end{array}\right\}$.

(C3) $M^{2}(0, T ; \mathbb{R}):=$

$\left\{\begin{array}{c}\varphi \text { : } \varphi \text { is jointly measurable processes such that }\|\varphi\|_{M^{2}}^{2}=E\left[\int_{0}^{T}\left|\varphi_{t}\right|^{2} d t\right] \\ <\infty \text {, and } \varphi_{t} \text { is } \mathscr{F}_{t}-\text { measurable, for any } t \in[0, T]\end{array}\right\}$.

(C3) $S_{\infty}(0, T ; \mathbb{R}):=$

$\left\{\begin{array}{c}\varphi: \varphi \text { is continuous processes with values in } \mathbb{R} \text { such that }\|\varphi\|_{S_{\infty}=}= \\ \sup _{\omega \in \Omega} \sup _{0 \leq t \leq T}\left|\varphi_{t}(\omega)\right|<\infty \text {, and } \varphi_{t} \text { is } \mathscr{F}_{t}-\text { measurable, for any } t \in[0, T]\end{array}\right\}$.

In order to study the qualitative properties of the solution to equations (1) and (2), we impose some assumptions on the coefficient functions, which will enable us to solve it.

(A1) Let $g: \Omega \times[0, T] \times \mathbb{R} \times \mathbb{R}^{d} \longrightarrow \mathbb{R}$ such that for any $(y, z) \in \mathbb{R} \times \mathbb{R}^{d}, g$ is $\mathscr{F}_{t}$-measurable and $L_{y}>0$ and $L_{z}>0$ are two constants. Then, for all $\left(t, y_{i}, z_{i}\right) \in[0, T] \times \mathbb{R} \times \mathbb{R}^{d}, i=1,2$,

$\left|g\left(t, y_{1}, z_{1}\right)-g\left(t, y_{2}, z_{2}\right)\right| \leq L_{y}\left|y_{1}-y_{2}\right|+L_{z}\left|z_{1}-z_{2}\right|$

(A2) $\xi \in L^{2}(\Omega, \mathscr{F}, \mathbb{P})$.

(A3) $g(\cdot, 0,0) \in M^{2}(0, T, \mathbb{R})$.

(A4) The obstacle $S \in S^{2}(0, T ; \mathbb{R})$.
(A5) $K_{t} \in L^{2}(\Omega, \mathscr{F}, \mathbb{P})$ is continuous and $S_{T}<\xi$.

(A6) $\xi$ is Malliavin differentiable and $\|D \xi\|_{\infty}:=$ $\sup _{0<t<T} \sup _{\omega \in \Omega}\left|D_{t} \xi\right|<\infty$, where $D_{t} \xi$ denotes the Malliavian derivative of $\xi$.

Lemma 1. It is known, since Pardoux and Peng in [1], that under the assumptions A1-A3, the BSDEs (1) have an adapted unique and square integrable solution $\left(Y_{t}, Z_{t}\right)_{t \in[0, T]}$ in $S^{2} \times M^{2}$. And arguing as in [15], one can show that the solution $\left(Y_{t}, Z_{t}\right)_{t \in[0, T]}$ is bounded. Moreover, we can get the following bounded for $Z$ :

$$
\|Z\|_{S_{\infty}} \leq\|D \xi\|_{S_{\infty}}^{2} e^{L_{y} T} .
$$

Lemma 2. It has been noticed that equation (2) has a unique solution in [6] under the assumptions A1-A5 and the following conditions:

(i) $\left(Y_{t}, Z_{t}, K_{t}\right) \in S^{2}(0, T ; \mathbb{R}) \times M^{2}\left(0, T ; \mathbb{R}^{d}\right) \times L^{2}(\Omega, P$, $\left.\mathbb{R}_{+}\right)$.

(ii) $Y_{t} \geq S_{t}, 0 \leq t \leq T$.

(iii) $K_{t}$ is continuous and increasing, $K_{0}=0$, and $\int_{0}^{T}\left(Y_{t}-S_{t}\right) d K_{t}=0$.

(iv) $Y_{t}=\xi+\int_{t}^{T} g\left(s, Y_{s}, Z_{s}\right) d s+K_{T}-K_{t}-\int_{t}^{T} Z_{s} d B_{s}, 0 \leq t$ $\leq T$.

In particular, since [6], one can easily see that $\left(Y_{t}, Z_{t}, K_{t}\right)$ is bounded.

\section{Averaging Principle for BSDEs}

In this section, we are going to investigate the averaging principle for the BSDEs under Lipschitz coefficients. Let us consider the standard form of equation (1):

$Y_{t}^{\varepsilon}=\xi+\varepsilon \int_{t}^{T} g\left(s, Y_{s}^{\varepsilon}, Z_{s}^{\varepsilon}\right) \mathrm{d} s-\sqrt{\varepsilon} \int_{t}^{T} Z_{s}^{\varepsilon} \mathrm{d} B_{s}$.

According to the second part, equation (5) also has an adapted unique and square integrable solution. We will examine whether the solution $Y_{t}^{\varepsilon}$ can be approximated to the solution process $\bar{Y}_{t}$ of the simplified equation:

$\bar{Y}_{t}=\xi+\varepsilon \int_{t}^{T} \bar{g}\left(\bar{Y}_{s}, \bar{Z}_{s}\right) \mathrm{d} s-\sqrt{\varepsilon} \int_{t}^{T} \bar{Z}_{s} \mathrm{~d} B_{s}$,

where $\left(\bar{Y}_{t}, \bar{Z}_{s}\right)$ has the same properties as $\left(Y_{s}^{\varepsilon}, Z_{s}^{\varepsilon}\right)$ and $\bar{g}: \mathbb{R} \times \mathbb{R}^{d} \longrightarrow \mathbb{R}$ is a measurable function satisfying $A 1$ and the additional inequalities.

(A7) For any $t \in\left[0, T_{1}\right] \subset[0, T]$, we have

$$
\begin{gathered}
\frac{1}{T_{1}-t} \int_{t}^{T_{1}}|g(s, Y, Z)-\bar{g}(Y, Z)|^{2} \mathrm{~d} s \\
\leq \varphi\left(T_{1}-t\right)\left(1+|Y|^{2}+|Z|^{2}\right),
\end{gathered}
$$

where $\varphi\left(T_{1}\right)$ is a bounded function. 
Theorem 1. Assume that conditions $A 1-A 3$ and $A 7$ are satisfied. For a given arbitrarily small number $\delta_{1}>0$, there exists $\varepsilon_{1} \in\left(0, \varepsilon_{0}\right]$ and $\beta \in(0,1)$ such that for all $\varepsilon \in\left(0, \varepsilon_{1}\right]$ having

$$
\sup _{T \varepsilon^{1-\beta} \leq t \leq T} \mathbb{E}\left|Y_{t}^{\mathcal{\varepsilon}}-\bar{Y}_{t}\right|^{2} \leq \delta_{1}
$$

In order to prove Theorem 1, we need the following Lemma 3.

Lemma 3. Under the assumptions of Theorem 1, let $[0, u] \subseteq[0, t] \subseteq[0, T]$ be arbitrary, and it holds that

$$
\mathbb{E} \int_{u}^{T}\left|Z_{s}^{\varepsilon}-\bar{Z}_{s}\right|^{2} \mathrm{~d} s \leq L \mathbb{E} \int_{u}^{T}\left|Y_{s}^{\varepsilon}-\bar{Y}_{s}\right|^{2} \mathrm{~d} s+C_{1}(T-u),
$$

where $L$ and $C_{1}$ are two constants depending on $u$.

Proof. By equations (5) and (6), we get

$$
\begin{aligned}
Y_{t}^{\varepsilon}-\bar{Y}_{t}= & \varepsilon \int_{u}^{T}\left(g\left(s, Y_{s}^{\varepsilon}, Z_{s}^{\varepsilon}\right)-\bar{g}\left(\bar{Y}_{t}, \bar{Z}_{s}\right)\right) \mathrm{d} s \\
& -\sqrt{\varepsilon} \int_{u}^{T}\left(Z_{s}^{\varepsilon}-\bar{Z}_{s}\right) \mathrm{d} B_{s} .
\end{aligned}
$$

Applying Itô's formula to $\left|Y_{t}^{\varepsilon}-\bar{Y}_{t}\right|^{2}$ and taking the mathematical expectation, we obtain

$$
\begin{aligned}
\varepsilon \mathbb{E} \int_{u}^{T}\left|Z_{s}^{\varepsilon}-\bar{Z}_{s}\right|^{2} \mathrm{~d} s+\mathbb{E}\left|Y_{t}^{\varepsilon}-\bar{Y}_{t}\right|^{2}= & 2 \varepsilon \mathbb{E} \int_{u}^{T}\left[\left(Y_{s}^{\varepsilon}-\bar{Y}_{s}\right)\left(g\left(s, Y_{s}^{\varepsilon}, Z_{s}^{\varepsilon}\right)-\bar{g}\left(\bar{Y}_{s}, \bar{Z}_{s}\right)\right)\right] \mathrm{d} s \\
\leq & 2 \varepsilon \mathbb{E} \int_{u}^{T}\left[\left(Y_{s}^{\varepsilon}-\bar{Y}_{s}\right)\left(g\left(s, Y_{s}^{\varepsilon}, Z_{s}^{\varepsilon}\right)-g\left(s, \bar{Y}_{s}, \bar{Z}_{s}\right)\right)\right] \mathrm{d} s \\
& +2 \varepsilon \mathbb{E} \int_{u}^{T}\left[\left(Y_{s}^{\varepsilon}-\bar{Y}_{s}\right)\left(g\left(s, \bar{Y}_{s}, \bar{Z}_{s}\right)-\bar{g}\left(\bar{Y}_{s}, \bar{Z}_{s}\right)\right)\right] \mathrm{d} s \\
= & B_{1}+B_{2} .
\end{aligned}
$$

For $B_{1}$, by using the condition $A 1$ and Young's inequality, for any $\theta>0$, we deduce that

$$
\begin{aligned}
B_{1} & \leq 2 \varepsilon \mathbb{E} \int_{u}^{T}\left[\left(Y_{s}^{\varepsilon}-\bar{Y}_{s}\right)\left(L_{y}\left|Y_{s}^{\varepsilon}-\bar{Y}_{s}\right|+L_{z}\left|Z_{s}^{\varepsilon}-\bar{Z}_{s}\right|\right)\right] \mathrm{d} s \\
& \leq 2 L_{y} \varepsilon \mathbb{E} \int_{u}^{T}\left|Y_{s}^{\varepsilon}-\bar{Y}_{s}\right|^{2} \mathrm{~d} s+2 L_{z} \varepsilon \mathbb{E} \int_{u}^{T}\left|Y_{s}^{\varepsilon}-\bar{Y}_{s}\right|\left|Z_{s}^{\varepsilon}-\bar{Z}_{s}\right| \mathrm{d} s \\
& \leq 2 L_{y} \varepsilon \mathbb{E} \int_{u}^{T}\left|Y_{s}^{\varepsilon}-\bar{Y}_{s}\right|^{2} \mathrm{~d} s+L_{z} \frac{1}{\theta} \varepsilon \mathbb{E} \int_{u}^{T}\left|Y_{s}^{\varepsilon}-\bar{Y}_{s}\right|^{2} \mathrm{~d} s+L_{z} \theta \varepsilon \mathbb{E} \int_{u}^{T}\left|Z_{s}^{\varepsilon}-\bar{Z}_{s}\right|^{2} \mathrm{~d} s \\
& =\left(2 L_{y}+\frac{L_{z}}{\theta}\right) \varepsilon \mathbb{E} \int_{u}^{T}\left|Y_{s}^{\varepsilon}-\bar{Y}_{s}\right|^{2} \mathrm{~d} s+L_{z} \theta \varepsilon \mathbb{E} \int_{u}^{T}\left|Z_{s}^{\varepsilon}-\bar{Z}_{s}\right|^{2} \mathrm{~d} s .
\end{aligned}
$$

For $B_{2}$, owing to the condition A2, Hölder's inequality and Young's inequality, it follows that

$$
\begin{aligned}
B_{2} & \leq 2 \varepsilon \mathbb{E}\left(\int_{u}^{T}\left|Y_{s}^{\varepsilon}-\bar{Y}_{s}\right|^{2} \mathrm{~d} s\right)^{1 / 2} \mathbb{E}\left(\int_{u}^{T}\left|g\left(s, \bar{Y}_{s}, \bar{Z}_{s}\right)-\bar{g}\left(\bar{Y}_{s}, \bar{Z}_{s}\right)\right|^{2} \mathrm{~d} s\right)^{1 / 2} \\
& \leq 2(T-u)^{1 / 2} \varepsilon \mathbb{E}\left(\int_{u}^{T}\left|Y_{s}^{\varepsilon}-\bar{Y}_{s}\right|^{2} \mathrm{~d} s\right)^{1 / 2} \mathbb{E}\left(\frac{1}{T-u} \int_{u}^{T}\left|g\left(s, \bar{Y}_{s}, \bar{Z}_{s}\right)-\bar{g}\left(\bar{Y}_{s}, \bar{Z}_{s}\right)\right|^{2} \mathrm{~d} s\right)^{1 / 2} \\
& \leq 2 \varepsilon \mathbb{E}\left((T-u) \int_{u}^{T}\left|Y_{s}^{\varepsilon}-\bar{Y}_{s}\right|^{2} \mathrm{~d} s\right)^{1 / 2}\left(\sup _{u \leq s \leq T}\left[\varphi^{2}(s-u)\right]\left(1+\sup _{u \leq s \leq T} \mathbb{E}\left(\left|\bar{Y}_{s}\right|^{2}\right)+\sup _{u \leq s \leq T} \mathbb{E}\left(\left|\bar{Z}_{s}\right|^{2}\right)\right)^{1 / 2}\right. \\
& \leq 2 C \varepsilon \mathbb{E}\left[(T-u) \int_{u}^{T}\left|Y_{s}^{\varepsilon}-\bar{Y}_{s}\right|^{2} \mathrm{~d} s\right]^{1 / 2} \\
& \leq C \varepsilon \mathbb{E}\left(\int_{u}^{T}\left|Y_{s}^{\varepsilon}-\bar{Y}_{s}\right|^{2} \mathrm{~d} s+T-u\right) \\
& =C \varepsilon \mathbb{E} \int_{u}^{T}\left|Y_{s}^{\varepsilon}-\bar{Y}_{s}\right|^{2} \mathrm{~d} s+C \varepsilon(T-u),
\end{aligned}
$$


where $\quad C=\left(\sup _{u \leq s \leq T}\left[\varphi^{2}(s-u)\right]\left(1+\sup _{u \leq s \leq T} \mathbb{E}\left(\left|\bar{Y}_{s}\right|^{2}\right)+\right.\right.$ $\left.\left.\sup _{u \leq s \leq T} \mathbb{E}\left(\left|\bar{Z}_{s}\right|^{2}\right)\right)\right)^{1 / 2}$. Now, we can choose $\theta=\left(1 / 2 L_{z}\right)>0$, then

$$
\begin{aligned}
\varepsilon \mathbb{E} \int_{u}^{T}\left|Z_{s}^{\varepsilon}-\bar{Z}_{s}\right|^{2} \mathrm{~d} s \leq & \left(2 L_{y}+\frac{L_{z}}{\theta}+C\right) \varepsilon \mathbb{E} \int_{u}^{T}\left|Y_{s}^{\varepsilon}-\bar{Y}_{s}\right|^{2} \mathrm{~d} s+L_{z} \theta \varepsilon \mathbb{E} \int_{u}^{T}\left|Z_{s}^{\varepsilon}-\bar{Z}_{s}\right|^{2} \mathrm{~d} s \\
& -\mathbb{E}\left|Y_{t}^{\varepsilon}-\bar{Y}_{t}\right|^{2}+C \varepsilon(T-u) \\
\leq & \left(2 L_{y}+2 L_{z}^{2}+C_{0}\right) \varepsilon \mathbb{E} \int_{u}^{T}\left|Y_{s}^{\varepsilon}-\bar{Y}_{s}\right|^{2} \mathrm{~d} s+\frac{1}{2} \varepsilon \mathbb{E} \int_{u}^{T}\left|Z_{s}^{\varepsilon}-\bar{Z}_{s}\right|^{2} \mathrm{~d} s \\
& +C \varepsilon(T-u) .
\end{aligned}
$$

Thus,

$$
\mathbb{E} \int_{u}^{T}\left|Z_{s}^{\varepsilon}-\bar{Z}_{s}\right|^{2} \mathrm{~d} s \leq L \mathbb{E} \int_{u}^{T}\left|Y_{s}^{\varepsilon}-\bar{Y}_{s}\right|^{2} \mathrm{~d} s+C_{1}(T-u),
$$

where $L=2\left(2 L_{y}+2 L_{z}^{2}+C_{0}\right), \quad C_{1}=2 C$. The proof is complete.
With the help of Lemma 3, now we can prove Theorem 1 by some conditions.

Proof. Using the elementary inequality and the isometry property, we derive that

$$
\begin{aligned}
\mathbb{E}\left|Y_{t}^{\varepsilon}-\bar{Y}_{t}\right|^{2}= & \mathbb{E}\left|\varepsilon \int_{u}^{T}\left[g\left(s, Y_{s}^{\varepsilon}, Z_{s}^{\varepsilon}\right)-\bar{g}\left(\bar{Y}_{s}, \bar{Z}_{s}\right)\right] \mathrm{d} s-\sqrt{\varepsilon} \int_{u}^{T}\left(Z_{s}^{\varepsilon}-\bar{Z}_{s}\right) \mathrm{d} B_{s}\right|^{2} \\
\leq & 2 \varepsilon^{2} \mathbb{E}\left|\int_{u}^{T}\left[g\left(s, Y_{s}^{\varepsilon}, Z_{s}^{\varepsilon}\right)-\bar{g}\left(\bar{Y}_{s}, \bar{Z}_{s}\right)\right] \mathrm{d} s\right|^{2}+2 \varepsilon \mathbb{E}\left|\int_{u}^{T}\left(Z_{s}^{\varepsilon}-\bar{Z}_{s}\right) \mathrm{d} B_{s}\right|^{2} \\
\leq & 4 \varepsilon^{2} \mathbb{E}\left|\int_{u}^{T}\left[g\left(s, Y_{s}^{\varepsilon}, Z_{s}^{\varepsilon}\right)-g\left(s, \bar{Y}_{s}, \bar{Z}_{s}\right)\right] \mathrm{d} s\right|^{2} \\
& +4 \varepsilon^{2} \mathbb{E}\left|\int_{u}^{T}\left[g\left(s, \bar{Y}_{s}, \bar{Z}_{s}\right)-\bar{g}\left(\bar{Y}_{s}, \bar{Z}_{s}\right)\right] \mathrm{d} s\right|^{2}+2 \varepsilon \mathbb{E} \int_{u}^{T}\left|Z_{s}^{\varepsilon}-\bar{Z}_{s}\right|^{2} \mathrm{~d} s \\
= & I_{1}+I_{2}+I_{3} .
\end{aligned}
$$

Applying Hölder's inequality and the assumption A1, we obtain

$$
\begin{aligned}
I_{1} & \leq 4(T-u) \varepsilon^{2} \mathbb{E} \int_{u}^{T}\left|g\left(s, Y_{s}^{\varepsilon}, Z_{s}^{\varepsilon}\right)-g\left(s, \bar{Y}_{s}, \bar{Z}_{s}\right)\right|^{2} \mathrm{~d} s \\
& \leq 4(T-u) \mathcal{E}^{2} \mathbb{E} \int_{u}^{T}\left(L_{y}\left|Y_{s}^{\varepsilon}-\bar{Y}_{s}\right|+L_{z}\left|Z_{s}^{\varepsilon}-\bar{Z}_{s}\right|\right)^{2} \mathrm{~d} s \\
& \leq 8 L_{y}^{2}(T-u) \varepsilon^{2} \mathbb{E} \int_{u}^{T}\left|Y_{s}^{\varepsilon}-\bar{Y}_{s}\right|^{2} \mathrm{~d} s+8 L_{z}^{2}(T-u) \mathcal{E}^{2} \mathbb{E} \int_{u}^{T}\left|Z_{s}^{\varepsilon}-\bar{Z}_{s}\right|^{2} \mathrm{~d} s .
\end{aligned}
$$

Then, together with Hölder's inequality and the assumption $A 7$, we get 


$$
\begin{aligned}
I_{2} & \leq 4(T-u) \varepsilon^{2} \mathbb{E} \int_{u}^{T}\left|g\left(s, \bar{Y}_{s}, \bar{Z}_{s}\right)-\bar{g}\left(\bar{Y}_{s}, \bar{Z}_{s}\right)\right|^{2} \mathrm{~d} s \\
& \leq 4(T-u) \varepsilon^{2} \mathbb{E} \int_{u}^{T}\left|g\left(s, \bar{Y}_{s}, \bar{Z}_{s}\right)-\bar{g}\left(\bar{Y}_{s}, \bar{Z}_{s}\right)\right|^{2} \mathrm{~d} s \\
& \leq 4(T-u)^{2} \varepsilon^{2} \sup _{u \leq s \leq T}\left[\varphi^{2}(s-u)\right]\left(1+\sup _{u \leq s \leq T} \mathbb{E}\left(\left|\bar{Y}_{s}\right|^{2}\right)+\sup _{u \leq s \leq T} \mathbb{E}\left(\left|\bar{Z}_{s}\right|^{2}\right)\right) \\
& =C_{2}(T-u)^{2} \varepsilon^{2},
\end{aligned}
$$

where $\quad C_{2}=4 \sup _{u \leq s \leq T}\left[\varphi^{2}(s-u)\right]\left(1+\sup _{u \leq s \leq T} \mathbb{E}\left(\left|\bar{Y}_{s}\right|^{2}\right)+\right.$ $\left.\sup _{u \leq s \leq T} \mathbb{E}\left(\left|\bar{Z}_{s}\right|^{2}\right)\right)$. According to Lemma 3, plug equations (16)-(18), and we refer that

$$
\begin{aligned}
\sup _{u \leq t \leq T} \mathbb{E}\left|Y_{t}^{\varepsilon}-\bar{Y}_{t}\right|^{2} \leq & 8 L_{y}^{2}(T-u) \varepsilon^{2} \sup _{u \leq t \leq T} \mathbb{E} \int_{u}^{T}\left|Y_{s}^{\varepsilon}-\bar{Y}_{s}\right|^{2} \mathrm{~d} s \\
& +\left(8 L_{z}^{2}(T-u) \varepsilon^{2}+2 \varepsilon\right) \sup _{u \leq t \leq T} \mathbb{E} \int_{u}^{T}\left|Z_{s}^{\varepsilon}-\bar{Z}_{s}\right|^{2} \mathrm{~d} s+C_{2}(T-u)^{2} \varepsilon^{2} \\
\leq & 8 L_{y}^{2}(T-u) \varepsilon^{2} \int_{u}^{T} \sup _{u \leq t \leq T} \mathbb{E}\left|Y_{s}^{\varepsilon}-\bar{Y}_{s}\right|^{2} \mathrm{~d} s \\
& +L\left(8 L_{z}^{2}(T-u) \varepsilon^{2}+2 \varepsilon\right) \int_{u \leq t \leq T}^{T} \sup _{u \leq t} \mathbb{E}\left|Y_{s}^{\varepsilon}-\bar{Y}_{s}\right|^{2} \mathrm{~d} s+C_{2}(T-u)^{2} \varepsilon^{2} \\
& +8 C_{1} L_{z}^{2}(T-u)^{2} \varepsilon^{2}+2 C_{1}(T-u) \varepsilon \\
\leq & \left(8 L_{y}^{2}(T-u) \varepsilon^{2}+8 L L_{z}^{2}(T-u) \varepsilon^{2}+2 L \varepsilon\right) \int_{u \leq s_{1} \leq s}^{T} \sup _{u}\left|Y_{s_{1}}^{\varepsilon}-\bar{Y}_{s_{1}}\right|^{2} \mathrm{~d} s \\
& +C_{3}(T-u)^{2} \varepsilon^{2}+2 C_{1}(T-u) \varepsilon,
\end{aligned}
$$

where $C_{3}=C_{2}+8 C_{1} L_{z}^{2}$. Thanks to Gronwall's inequality, we obtain

$$
\begin{aligned}
\sup _{u \leq t \leq T} \mathbb{E}\left|Y_{t}^{\varepsilon}-\bar{Y}_{t}\right|^{2} \leq & \left(C_{3}(T-u)^{2} \varepsilon^{2}+2 C_{1}(T-u) \varepsilon\right) \\
& \cdot e^{(T-u)\left(8 L_{y}^{2}(T-u) \varepsilon^{2}+8 L L_{z}^{2}(T-u) \varepsilon^{2}+2 L \varepsilon\right)} .
\end{aligned}
$$

Obviously, the above estimate implies that there exists $\beta \in(0,1)$, for every $t \in\left[T \varepsilon^{1-\beta}, T\right]$,

$$
\sup _{T \varepsilon^{1-\beta} \leq t \leq T} \mathbb{E}\left|Y_{t}^{\varepsilon}-\bar{Y}_{t}\right|^{2} \leq C_{4} \varepsilon
$$

in which $\quad C_{4}=\left(C_{3} T^{2}\left(1-\varepsilon^{1-\beta}\right)^{2} \varepsilon+2 C_{1} T\left(1-\varepsilon^{1-\beta}\right)\right.$ $e^{T\left(1-\varepsilon^{1-\beta}\right)\left(8 L_{y}^{2} T\left(1-\varepsilon^{1-\beta}\right) \varepsilon^{2}+8 L L_{z}^{2} T\left(1-\varepsilon^{1-\beta}\right) \varepsilon^{2}+2 L \varepsilon\right)}$ is a constant.
Consequently, for any number $\delta_{1}>0$, we can choose $\varepsilon_{1} \in\left(0, \varepsilon_{0}\right]$ making

$$
\sup _{T \varepsilon^{1-\beta} \leq t \leq T} \mathbb{E}\left|Y_{t}^{\varepsilon}-\bar{Y}_{t}\right|^{2} \leq \delta_{1}
$$

where $\varepsilon \in\left(0, \varepsilon_{1}\right]$. Hence, the proof is complete.

\section{Averaging Principle for RBSDEs with a Barrier}

In this section, we will continue to study the averaging principle for the RBSDEs under Lipschitz condition. Firstly, let us consider the standard form of equation (2):

$$
\begin{aligned}
Y_{t}^{\varepsilon} & =\xi+\varepsilon \int_{t}^{T} g\left(s, Y_{s}^{\varepsilon}, Z_{s}^{\varepsilon}\right) \mathrm{d} s+K_{T}-K_{t}-\sqrt{\varepsilon} \int_{t}^{T} Z_{s}^{\varepsilon} \mathrm{d} B_{s} \\
Y_{t}^{\varepsilon} & \geq S_{t} \\
K_{0} & =0 \\
\int_{0}^{T}\left(Y_{t}^{\varepsilon}-S_{t}\right) \mathrm{d} K_{t} & =0
\end{aligned}
$$


In fact, according to Section 2 (Lemma 1), it is easy to find that equation (23) also has a unique solution. Then, we consider the simplified system:

$$
\begin{aligned}
\bar{Y}_{t} & =\xi+\varepsilon \int_{t}^{T} \bar{g}\left(\bar{Y}_{s}, \bar{Z}_{s}\right) \mathrm{d} s+\bar{K}_{T}-\bar{K}_{t}-\sqrt{\varepsilon} \int_{t}^{T} \bar{Z}_{s} \mathrm{~d} B_{s}, \\
\bar{Y}_{t} & \geq S_{t}, \\
\bar{K}_{0} & =0, \\
\int_{0}^{T}\left(\bar{Y}_{t}-S_{t}\right) \mathrm{d} \bar{K}_{t} & =0,
\end{aligned}
$$

where $\bar{K}_{t}$ is a continuous function satisfying A5 and Lemma 1.

Theorem 2. Assume that conditions A1-A5 are satisfied. For a given arbitrarily small number $\delta_{2}>0$, there exists $\varepsilon_{1} \in\left(0, \varepsilon_{0}\right]$ and $\beta \in(0,1)$ such that for all $\varepsilon \in\left(0, \varepsilon_{1}\right]$,

$$
\sup _{T \varepsilon^{1-\beta} \leq t \leq T} \mathbb{E}\left|Y_{t}^{\varepsilon}-\bar{Y}_{t}\right|^{2} \leq \delta_{2} \text {. }
$$

Before giving the proof of Theorem 2, we need some Lemmas as follows.
Lemma 4. Under the assumptions of Theorem 2, let $[0, u] \subseteq[0, t] \subseteq[0, T]$ be arbitrary, for any $t \in[0, u]$, there exists a constant $L$ such that

$$
\mathbb{E} \int_{u}^{T}\left|Z_{s}^{\varepsilon}-\bar{Z}_{s}\right|^{2} \mathrm{~d} s \leq L \mathbb{E} \int_{u}^{T}\left|Y_{s}^{\varepsilon}-\bar{Y}_{s}\right|^{2} \mathrm{~d} s+C_{1}(T-u) .
$$

Proof. If equations (23) and (24) are satisfied, according to the property of $K_{T}, \bar{K}_{T}$ in $\mathrm{A} 5$, then applying It $\widehat{o}$ 's formula to $\left|Y_{t}^{\varepsilon}-\bar{Y}_{t}\right|^{2}$ and taking the mathematical expectation, we obtain that

$$
\begin{aligned}
\varepsilon \mathbb{E} \int_{u}^{T}\left|Z_{s}^{\varepsilon}-\bar{Z}_{s}\right|^{2} \mathrm{~d} s+\mathbb{E}\left|Y_{t}^{\varepsilon}-\bar{Y}_{t}\right|^{2}= & 2 \varepsilon \mathbb{E} \int_{u}^{T}\left[\left(Y_{s}^{\varepsilon}-\bar{Y}_{s}\right)\left(g\left(s, Y_{s}^{\varepsilon}, Z_{s}^{\varepsilon}\right)-\bar{g}\left(\bar{Y}_{s}, \bar{Z}_{s}\right)\right)\right] \mathrm{d} s \\
& +2 \mathbb{E} \int_{u}^{T}\left(Y_{s}^{\varepsilon}-\bar{Y}_{s}\right) \mathrm{d}\left(K_{s}-\bar{K}_{s}\right) .
\end{aligned}
$$

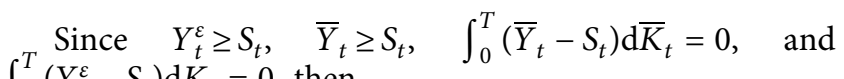
$\int_{0}^{T}\left(Y_{t}^{\varepsilon}-S_{t}\right) \mathrm{d} K_{t}=0$, then

$$
\begin{aligned}
2 \mathbb{E} \int_{u}^{T}\left(Y_{s}^{\varepsilon}-\bar{Y}_{s}\right) \mathrm{d}\left(K_{s}-\bar{K}_{s}\right)= & 2 \mathbb{E} \int_{u}^{T}\left(Y_{s}^{\varepsilon}-S_{t}\right) \mathrm{d}\left(K_{s}-\bar{K}_{s}\right) \\
& -2 \mathbb{E} \int_{u}^{T}\left(\bar{Y}_{s}-S_{t}\right) \mathrm{d}\left(K_{s}-\bar{K}_{s}\right) \\
= & 2 \mathbb{E} \int_{u}^{T}\left(Y_{s}^{\varepsilon}-S_{t}\right) \mathrm{d} K_{s}-2 \mathbb{E} \int_{u}^{T}\left(Y_{s}^{\varepsilon}-S_{t}\right) \mathrm{d} \bar{K}_{s} \\
& -2 \mathbb{E} \int_{u}^{T}\left(\bar{Y}_{s}-S_{t}\right) \mathrm{d} K_{s}+2 \mathbb{E} \int_{u}^{T}\left(\bar{Y}_{s}-S_{t}\right) \mathrm{d} \bar{K}_{s} \\
\leq & 2 \mathbb{E} \int_{u}^{T}\left(Y_{s}^{\varepsilon}-S_{t}\right) \mathrm{d} K_{s}+2 \mathbb{E} \int_{u}^{T}\left(\bar{Y}_{s}-S_{t}\right) \mathrm{d} \bar{K}_{s} \\
\leq & 2 \mathbb{E} \int_{0}^{T}\left(Y_{s}^{\varepsilon}-S_{t}\right) \mathrm{d} K_{s}+2 \mathbb{E} \int_{0}^{T}\left(\bar{Y}_{s}-S_{t}\right) \mathrm{d} \bar{K}_{s} \\
= & 0 .
\end{aligned}
$$


It follows that

$$
\begin{aligned}
\varepsilon \mathbb{E} & \int_{u}^{T}\left|Z_{s}^{\varepsilon}-\bar{Z}_{s}\right|^{2} \mathrm{~d} s+\mathbb{E}\left|Y_{t}^{\varepsilon}-\bar{Y}_{t}\right|^{2} \\
\quad & =2 \varepsilon \mathbb{E} \int_{u}^{T}\left[\left(Y_{s}^{\varepsilon}-\bar{Y}_{s}\right)\left(g\left(s, Y_{s}^{\varepsilon}, Z_{s}^{\varepsilon}\right)-\bar{g}\left(\bar{Y}_{s}, \bar{Z}_{s}\right)\right)\right] \mathrm{d} s .
\end{aligned}
$$

Recalling equations (11)-(14), it is easy to obtain the result of Lemma 3.

Lemma 5. Under the assumptions of Theorem 2, let $u \in[0, T]$ be arbitrary, for any $[0, u] \subseteq[0, t] \subseteq[0, T]$, it holds that

$$
\begin{aligned}
\mathbb{E}\left|K_{t}-\bar{K}_{t}\right|^{2} \mathrm{~d} s \leq & 3 \varepsilon^{2}\left(4 L_{y}^{2}(T-u)+4 L L_{z}^{2}(T-u)+\frac{L}{\varepsilon}\right) \mathbb{E} \int_{u}^{T}\left|Y_{s}^{\varepsilon}-\bar{Y}_{s}\right|^{2} \mathrm{~d} s \\
& +C_{6}(T-u)^{2} \varepsilon^{2}+3 C_{1}(T-u) \varepsilon,
\end{aligned}
$$

where $C_{3}$ is a constant.

Proof. By equations (23) and (24), we obtain that

$$
\left|K_{t}-\bar{K}_{t}\right|=\left|\varepsilon \int_{u}^{T}\left[g\left(s, Y_{s}^{\varepsilon}, Z_{s}^{\varepsilon}\right)-\bar{g}\left(\bar{Y}_{s}, \bar{Z}_{s}\right)\right] \mathrm{d} s-\sqrt{\varepsilon} \int_{u}^{T}\left(Z_{s}^{\varepsilon}-\bar{Z}_{s}\right) \mathrm{d} B_{s}+K_{T}-\bar{K}_{T}\right|
$$

Applying Hölder's inequality, the isometry property to $\left|K_{t}^{\varepsilon}-\bar{K}_{t}\right|$ and taking the mathematical expectation, it follows that

$$
\begin{aligned}
\mathbb{E}\left|K_{t}-\bar{K}_{t}\right|^{2} \leq & 3 \varepsilon^{2} \mathbb{E}\left|\int_{u}^{T}\left[g\left(s, Y_{s}^{\varepsilon}, Z_{s}^{\varepsilon}\right)-\bar{g}\left(\bar{Y}_{s}, \bar{Z}_{s}\right)\right] \mathrm{d} s\right|^{2}+3 \varepsilon \mathbb{E}\left|\int_{u}^{T}\left(Z_{s}^{\varepsilon}-\bar{Z}_{s}\right) \mathrm{d} B_{s}\right|^{2} \\
\leq & 3 \varepsilon^{2} \mathbb{E}\left|\int_{u}^{T}\left[g\left(s, Y_{s}^{\varepsilon}, Z_{s}^{\varepsilon}\right)-\bar{g}\left(\bar{Y}_{s}, \bar{Z}_{s}\right)\right] \mathrm{d} s\right|^{2}+3 \varepsilon \mathbb{E} \int_{u}^{T}\left|Z_{s}^{\varepsilon}-\bar{Z}_{s}\right|^{2} \mathrm{~d} s \\
\leq & 3(T-u) \varepsilon^{2} \mathbb{E} \int_{u}^{T}\left|g\left(s, Y_{s}^{\varepsilon}, Z_{s}^{\varepsilon}\right)-\bar{g}\left(\bar{Y}_{s}, \bar{Z}_{s}\right)\right|^{2} \mathrm{~d} s+3 \varepsilon \mathbb{E} \int_{u}^{T}\left|Z_{s}^{\varepsilon}-\bar{Z}_{s}\right|^{2} \mathrm{~d} s \\
\leq & 6(T-u) \varepsilon^{2} \mathbb{E} \int_{u}^{T}\left|g\left(s, Y_{s}^{\varepsilon}, Z_{s}^{\varepsilon}\right)-g\left(s, \bar{Y}_{s}, \bar{Z}_{s}\right)\right|^{2} \mathrm{~d} s \\
& +6(T-u)^{2} \varepsilon^{2} \mathbb{E} \frac{1}{T-u} \int_{u}^{T}\left|g\left(s, \bar{Y}_{s}, \bar{Z}_{s}\right)-\bar{g}\left(\bar{Y}_{s}, \bar{Z}_{s}\right)\right|^{2} \mathrm{~d} s \\
& +3 \varepsilon \mathbb{E} \int_{u}^{T}\left|Z_{s}^{\varepsilon}-\bar{Z}_{s}\right|^{2} \mathrm{~d} s,
\end{aligned}
$$

where we used the fact that $\mathbb{E}\left|K_{T}-\bar{K}_{T}\right|^{2}=0$. Owing to the assumptions $\mathrm{A} 1$ and $\mathrm{A} 7$, we have 


$$
\begin{aligned}
\mathbb{E}\left|K_{t}-\bar{K}_{t}\right|^{2} \leq & 6(T-u) \varepsilon^{2} \mathbb{E} \int_{u}^{T}\left(L_{y}\left|Y_{s}^{\varepsilon}-\bar{Y}_{s}\right|+L_{z}\left|Z_{s}^{\varepsilon}-\bar{Z}_{s}\right|\right)^{2} \mathrm{~d} s \\
& +6(T-u)^{2} \varepsilon^{2} \sup _{u \leq s \leq T}\left[\varphi^{2}(s)\right]\left(1+\sup _{u \leq s \leq T} \mathbb{E}\left(\left|\bar{Y}_{s}\right|^{2}\right)+\sup _{u \leq s \leq T} \mathbb{E}\left(\left|\bar{Z}_{s}\right|^{2}\right)\right. \\
& +3 \varepsilon \mathbb{E} \int_{u}^{T}\left|Z_{s}^{\varepsilon}-\bar{Z}_{s}\right|^{2} \mathrm{~d} s \\
\leq & 12 L_{y}^{2}(T-u) \varepsilon^{2} \mathbb{E} \int_{u}^{T}\left|Y_{s}^{\varepsilon}-\bar{Y}_{s}\right|^{2} \mathrm{~d}+12 L_{z}^{2}(T-u) \varepsilon^{2} \mathbb{E} \int_{u}^{T}\left|Z_{s}^{\varepsilon}-\bar{Z}_{s}\right|^{2} \mathrm{~d} s \\
& +C_{5}(T-u)^{2} \varepsilon^{2}+3 \varepsilon \mathbb{E} \int_{u}^{T}\left|Z_{s}^{\varepsilon}-\bar{Z}_{s}\right|^{2} \mathrm{~d} s,
\end{aligned}
$$

where $\quad C_{5}=\sup _{u \leq s \leq T}\left[\varphi^{2}(s)\right]\left(1+\sup _{u \leq s \leq T} \mathbb{E}\left(\left|\bar{Y}_{s}\right|^{2}\right)+\right.$

$\sup _{u \leq s \leq T} \mathbb{E}\left(\left|\bar{Z}_{s}\right|^{2}\right)$. Meanwhile, from Lemma 4, we get

$$
\begin{aligned}
\mathbb{E}\left|K_{t}-\bar{K}_{t}\right|^{2} \leq & \left(12 L_{y}^{2}(T-u) \varepsilon^{2}+12 L L_{z}^{2}(T-u) \varepsilon^{2}+3 L \varepsilon\right) \mathbb{E} \int_{u}^{T}\left|Y_{s}^{\varepsilon}-\bar{Y}_{s}\right|^{2} \mathrm{~d} s+C_{5}(T-u)^{2} \varepsilon^{2} \\
& +12 C_{1} L_{z}^{2}(T-u)^{2} \varepsilon^{2}+3 C_{1}(T-u) \varepsilon \\
\leq & 3 \varepsilon^{2}\left(4 L_{y}^{2}(T-u)+4 L L_{z}^{2}(T-u)+\frac{L}{\varepsilon}\right) \mathbb{E} \int_{u}^{T}\left|Y_{s}^{\varepsilon}-\bar{Y}_{s}\right|^{2} \mathrm{~d} s+C_{6}(T-u)^{2} \varepsilon^{2} \\
& +3 C_{1}(T-u) \varepsilon
\end{aligned}
$$

where $C_{6}=C_{5}+12 C_{1} L_{z}^{2}$. The proof is completed.

Proof. Together with the elementary equality and the isometry property, we deduce that

Motivated by the above results, now we can prove Theorem 2.

$$
\begin{aligned}
\sup _{u \leq t \leq T} \mathbb{E}\left|Y_{t}^{\varepsilon}-\bar{Y}_{t}\right|^{2}= & \sup _{u \leq t \leq T} \mathbb{E}\left|\varepsilon \int_{u}^{T}\left[g\left(s, Y_{s}^{\varepsilon}, Z_{s}^{\varepsilon}\right)-\bar{g}\left(\bar{Y}_{s}, \bar{Z}_{s}\right)\right] \mathrm{d} s-\sqrt{\varepsilon} \int_{u}^{T}\left(Z_{s}^{\varepsilon}-\bar{Z}_{s}\right) \mathrm{d} B_{s}+K_{T}-\bar{K}_{T}+K_{t}-\bar{K}_{t}\right|^{2} \\
\leq & 4 \varepsilon^{2} \sup _{u \leq t \leq T} \mathbb{E}\left|\int_{u}^{T}\left[g\left(s, Y_{s}^{\varepsilon}, Z_{s}^{\varepsilon}\right)-\bar{g}\left(\bar{Y}_{s}, \bar{Z}_{s}\right)\right] \mathrm{d} s\right|^{2} \\
& +4 \varepsilon \sup _{u \leq t \leq T} \mathbb{E}\left|\int_{u}^{T}\left(Z_{s}^{\varepsilon}-\bar{Z}_{s}\right) \mathrm{d} B_{s}\right|^{2}+4 \sup _{u \leq t \leq T} \mathbb{E}\left|K_{t}-\bar{K}_{t}\right|^{2} \\
= & J_{1}+J_{2}+J_{3} .
\end{aligned}
$$

Recalling (17) and (18), we have

$$
\begin{aligned}
J_{1} \leq & 16(T-u) \varepsilon^{2}\left(L_{y}^{2}+L L_{z}^{2}\right) \\
& \cdot \sup _{u \leq t \leq T} \mathbb{E} \int_{u}^{T}\left|Y_{s}^{\varepsilon}-\bar{Y}_{s}\right|^{2} \mathrm{~d} s+2 C_{2}(T-u)^{2} \varepsilon^{2} \\
& +16 C_{1} L_{z}^{2}(T-u)^{2} \varepsilon^{2} .
\end{aligned}
$$

For $J_{2}$, combining the isometry property with Lemma 4 produces

$$
\begin{aligned}
J_{2} \leq & 4 \varepsilon \sup _{u \leq t \leq T} \mathbb{E} \int_{u}^{T}\left|Z_{s}^{\varepsilon}-\bar{Z}_{s}\right|^{2} \mathrm{~d} s \leq 4 L \varepsilon \\
& \sup _{u \leq t \leq T} \mathbb{E} \int_{u}^{T}\left|Y_{s}^{\varepsilon}-\bar{Y}_{s}\right|^{2} \mathrm{~d} s+4 C_{1}(T-u) \varepsilon .
\end{aligned}
$$


By virtue of Lemma 5, we obtain

$$
\begin{aligned}
J_{3} \leq & 12 \varepsilon^{2}\left(4 L_{y}^{2}(T-u)+4 L L_{z}^{2}(T-u)+\frac{L}{\varepsilon}\right) \sup _{u \leq t \leq T} \mathbb{E} \int_{u}^{T}\left|Y_{s}^{\varepsilon}-\bar{Y}_{s}\right|^{2} \mathrm{~d} s+4 C_{6}(T-u)^{2} \varepsilon^{2} \\
& +12 C_{1}(T-u) \varepsilon .
\end{aligned}
$$

Now, plug equations (36)-(38) into (35) for any $u \leq t \leq T$, and we get

$$
\begin{aligned}
\sup _{u \leq t \leq T} \mathbb{E}\left|Y_{t}^{\varepsilon}-\bar{Y}_{t}\right|^{2} \leq & \left(64 L_{y}^{2}(T-u)+64 L L_{z}^{2}(T-u)+\frac{16 L}{\varepsilon}\right) \varepsilon^{2} \sup _{u \leq t \leq T} \mathbb{E} \int_{u}^{T}\left|Y_{s}^{\varepsilon}-\bar{Y}_{s}\right|^{2} \mathrm{~d} s \\
& +C_{7}(T-u)^{2} \varepsilon^{2}+16 C_{1}(T-u) \varepsilon \\
\leq & \left(64 L_{y}^{2} T+64 L L_{z}^{2}(T-u)+\frac{16 L}{\varepsilon}\right) \varepsilon^{2} \int_{u}^{T} \sup _{u \leq t \leq T} \mathbb{E}\left|Y_{s_{1}}^{\varepsilon}-\bar{Y}_{s_{1}}\right|^{2} \mathrm{~d} s \\
& +C_{7}(T-u)^{2} \varepsilon^{2}+16 C_{1}(T-u) \varepsilon,
\end{aligned}
$$

where $C_{7}=16 C_{1} L_{z}^{2}+2 C_{2}+4 C_{6}$. In terms of Gronwall's inequality, we obtain

$$
\begin{aligned}
\sup _{u \leq t \leq T} \mathbb{E}\left|Y_{t}^{\varepsilon}-\bar{Y}_{t}\right|^{2} \leq & \left(C_{7}(T-u)^{2} \varepsilon^{2}+16 C_{1}(T-u) \varepsilon\right) \\
& \cdot e^{64 L_{y}^{2}(T-u)^{2} \varepsilon^{2}+64 L L_{z}^{2}(T-u)^{2} \varepsilon^{2}+16(T-u) L \varepsilon} .
\end{aligned}
$$

Similarly, we can choose $\beta \in(0,1)$ and $L>0$ such that for every $t \in\left[T \varepsilon^{1-\beta}, T\right]$, then

$$
\sup _{T \varepsilon^{1-\beta} \leq t \leq T} \mathbb{E}\left|Y_{t}^{\varepsilon}-\bar{Y}_{t}\right|^{2} \leq C_{8} \varepsilon^{1-\beta},
$$

where $\quad C_{8}=\left(C_{7} T^{2}\left(1-\varepsilon^{1-\beta}\right)^{2} \varepsilon+16 C_{1} T\left(1-\varepsilon^{1-\beta}\right)\right)$ $e^{\left(T^{2}\left(1-\varepsilon^{1-\beta}\right)^{2} \varepsilon^{2}\right)\left(64 L_{y}^{2}+64 L L_{z}^{2}+\left(16 L / T \varepsilon\left(1-\varepsilon^{1-\beta}\right)\right)\right)}$. Indeed, for any number $\delta_{2}$, we can select $\varepsilon_{1} \in\left(0, \varepsilon_{0}\right]$ such that for every $\varepsilon \in\left(0, \varepsilon_{1}\right]$ and $t \in\left[T \varepsilon^{1-\beta}, T\right]$, the inequality

$$
\sup _{T \varepsilon^{1-\beta} \leq t \leq T} \mathbb{E}\left|Y_{t}^{\varepsilon}-\bar{Y}_{t}\right|^{2} \leq \delta_{2}
$$

holds. Hence, it is proved.

\section{Examples}

Now, two examples will be presented to illustrate the procedure of the obtained theory.

Example 1. Consider the following BSDE for any $t \in[0, T]$ :

$$
Y^{\varepsilon}=\xi+\varepsilon \int_{t}^{T}\left(Y^{\varepsilon} \sin ^{2} s-Z^{\varepsilon} \cos ^{2} s\right) \mathrm{d} s-\sqrt{\varepsilon} \int_{t}^{T} Z^{\varepsilon} \mathrm{d} B_{s},
$$

where $g\left(t, Y^{\varepsilon}, Z^{\varepsilon}\right)=Y^{\varepsilon} \sin ^{2} s-Z^{\varepsilon} \cos ^{2} s$ and

$$
\begin{aligned}
\bar{g}\left(Y^{\varepsilon}, Z^{\varepsilon}\right) & =\frac{1}{T-t} \int_{t}^{T} g\left(s, Y^{\varepsilon}, Z^{\varepsilon}\right) \mathrm{d} s \\
& =\frac{1}{T-t} \int_{t}^{T}\left(Y^{\varepsilon} \sin ^{2} s-Z^{\varepsilon} \cos ^{2} s\right) \mathrm{d} s \\
& =\frac{Y^{\varepsilon}}{2}\left(1+\frac{\sin 2 t}{2(\pi-t)}\right)-\frac{Z^{\varepsilon}}{2}\left(1-\frac{\sin 2 t}{2(\pi-t)}\right),
\end{aligned}
$$

and we can define a new simplified BSDE as

$$
\bar{Y}=\xi+\varepsilon \int_{t}^{T} \bar{g}(\bar{Y}, \bar{Z}) \mathrm{d} s-\sqrt{\varepsilon} \int_{t}^{T} \bar{Z} \mathrm{~d} B_{s} .
$$

It is easy to check that assumptions A1-A3 and A7 are satisfied for $g$ and $\bar{g}$; therefore, Theorem 1 and Lemma 3 are satisfied; that is,

$$
\sup _{T \varepsilon^{1-\beta} \leq t \leq T} \mathbb{E}\left|Y_{t}^{\varepsilon}-\bar{Y}_{t}\right|^{2} \leq \delta_{1} .
$$

Example 2. we consider the following RBSDE for any $t \in[0, T]:$

$$
\begin{aligned}
Y^{\varepsilon}= & \xi+\varepsilon \int_{t}^{T}\left(Y^{\varepsilon} \cos ^{2} s-Z^{\varepsilon} \sin ^{2} s\right) \mathrm{d} s \\
& -\sqrt{\varepsilon} \int_{t}^{T} \frac{1}{2} Z^{\varepsilon} \mathrm{d} B_{s}+K_{T}-K_{t}
\end{aligned}
$$

where $g\left(t, Y^{\varepsilon}, Z^{\varepsilon}\right)=Y^{\varepsilon} \cos ^{2} s-Z^{\varepsilon} \sin ^{2} s, S_{t}=0$, for any $t \in[0, T]$ and 


$$
\begin{aligned}
\bar{g}\left(Y^{\varepsilon}, Z^{\varepsilon}\right) & =\frac{1}{T-t} \int_{t}^{T} g\left(s, Y^{\varepsilon}, Z^{\varepsilon}\right) \mathrm{d} s \\
& =\frac{1}{\pi-t} \int_{t}^{\pi}\left(Y^{\varepsilon} \cos ^{2} s-Z^{\varepsilon} \sin ^{2} s\right) \mathrm{d} s \\
& =\frac{Y^{\varepsilon}}{2}\left(1-\frac{\sin 2 t}{2(\pi-t)}\right)-\frac{Z^{\varepsilon}}{2}\left(1+\frac{\sin 2 t}{2(\pi-t)}\right),
\end{aligned}
$$

and the simplified system can be obtained as

$$
\bar{Y}_{t}=\xi+\varepsilon \int_{t}^{T} \bar{g}\left(\bar{Y}_{s}, \bar{Z}_{s}\right) \mathrm{d} s-\sqrt{\varepsilon} \int_{t}^{T} \frac{1}{2} \bar{Z}_{s} \mathrm{~d} B_{s}+\bar{K}_{T}-\bar{K}_{t},
$$

where $\bar{S}_{t}=0$ for any $t \in[0, T]$. We can check that all the assumptions A1-A5 and A7 hold for $g$ and $\bar{g}$; therefore, Theorem 2 and Lemmas 4 and 5 are satisfied; that is,

$$
\sup _{T \mathcal{E}^{1-\beta} \leq t \leq T} \mathbb{E}\left|Y_{t}^{\mathcal{\varepsilon}}-\bar{Y}_{t}\right|^{2} \leq \delta_{2} \text {. }
$$

\section{Conclusions}

Backward stochastic differential equations are widely used in finance and optimal control problems. The contribution of this paper is that, different from traditional stochastic differential equations, the averaging principle of backward stochastic differential equations under Lipschitz condition is given.

\section{Data Availability}

The data used in this article are available from the authors upon request

\section{Conflicts of Interest}

The authors declare that they have no conflicts of interest.

\section{References}

[1] E. Pardoux and S. G. Peng, "Adapted solution of a backward stochastic differential equation," Systems \& Control Letters, vol. 14, no. 1, pp. 55-61, 1990.

[2] X. Mao, "Adapted solutions of backward stochastic differential equations with non-Lipschitz coefficients," Stochastic Processes and Their Applications, vol. 58, no. 2, pp. 281-292, 1995.

[3] Y. Wang and X. Wang, "Adapted solutions of backward SDE with non-Lipschitz coefficients," Chinese Journal of Applied Probability and Statisties, vol. 19, pp. 245-251, 2003.

[4] J. P. Lepeltier and J. San Martin, "Backward stochastic differential equations with continuous coefficient," Statistics \& Probability Letters, vol. 32, no. 4, pp. 425-430, 1997.

[5] K. Bahlali, "Backward stochastic differential equations with locally Lipschitz coefficient," Comptes Rendus de l'Académie des Sciences - Series I - Mathematics mie des Science-Series I-Mathematics, vol. 333, no. 5, pp. 481-486, 2001.

[6] N. EI Karoui, C. Kapoudjian, E. Pardoux, S. Peng, and M. C. Quenez, "Reflected solutions of backward SDE and related obstacle problems for PDEs," The Annals of Probability, vol. 25, no. 2, pp. 702-737, 1997.

[7] A. Matoussi, "Reflected solutions of backward stochastic differential equations with continuous coefficient," Statistics \& Probability Letters, vol. 34, no. 4, pp. 347-354, 1997.

[8] S. Hamadene, "Reflected BSDEs's with discontinuous barrier and application," Stochastics: An International Journal of Probability and Stochastic Processes, vol. 74, no. 3, pp. 571-596, 2002.

[9] J. P. Lepeltier and M. Xu, "Penalization method for reflected backward stochastic differential equations with one r.c.l.1. barrier," Statistics \& Probability Letters, vol. 75, no. 1, pp. 58-66, 2005.

[10] J. P. Lepeltier, A. Matoussi, and M. Xu, Reflected BSDEs under Momotonicity and General Inceasing Growth Conditions, Preprint Universite du Maine, France, 2004.

[11] R. Z. Khas'minskii, "A limit theorem for the solution of differential equations with random right-hand sides," Theory of Probability and Its Application, vol. 11, no. 3, pp. 390-406, 1966.

[12] R. Z. Khas'minskii, "On the principle of averaging the it stochastic differential equations," Kybernetika (Prague), vol. 4, pp. 260-279, 1968.

[13] I. M. Stoyanov and D. D. Bainov, "The averaging method for a class of stochastic differential equations," Ukrainian Mathematics Journal, vol. 26, pp. 186-194, 1974.

[14] L. N'Gorn and M. N'Zi, “Averaging principle for multivalued stochastic differential equations," Random Operators and Stochastic Equations, vol. 9, pp. 399-407, 2001.

[15] S. Hamadene, "Quations diff rentielles stochastiques retrogrades: Le cas localement lipschitizien," Annales De L Institut Henri Poincar Probabilites et Statistiques, vol. 32, no. 5, pp. 645-659, 1996. 